\title{
CFD BASED AERODYNAMIC STUDY TO DISCRETE OPTIMIZATION OF BRIDGE CROSS SECTIONS
}

\author{
Adelino V. Lopes ${ }^{1}$, Carlos D. P. C. Gomes ${ }^{1}$, and Luís M.C. Simões ${ }^{1}$ \\ ${ }^{1}$ Department of Civil Engineering \\ Faculty of Sciences and Technology of the University of Coimbra, Coimbra, Portugal \\ \{avlopes, diugo, lcsimoes\}@dec.uc.pt
}

Keywords: CFD, FVM, FOM, bridges, Scanlan Model, Aerodynamic coefficients, Flutter, Improving cross section.

\begin{abstract}
Aerodynamic instability of bridges should be one of the most concerns for bridge designers. Along with all studies about aerodynamic studies, a few ones are related to improvements of bridge cross sections. Among them, strategies such as grating, edge plates, edge fairing plates, side plates, baffle plates or flaps has been tested. The aim of this study is associated with the efficiency of using some based lateral inside appendages with the purpose of improving the aerodynamic characteristics of a $\Pi$ cross section $(B / D=6)$. It is turned to the Scanlan model, namely to the $A * 2$ coefficient, with the aim of evaluating the aerodynamic efficiency of the cross section. In order to determine the fluid flow around the obstacle, it is used a numerical algorithm of computational fluid dynamics based on the Finite Volume Method (FVM). Additionally, Forced Oscillation Method (FOM) is adopted for evaluating aeroelastic coefficients.
\end{abstract}




\section{INTRODUCTION}

Wind action is one of the most determining factors for the safety of large and flexible structures. As it is well known, since the famous Tacoma Narrows Bridge failure, in 1940, the design of long span cable-stayed and suspension bridges requires careful study of their aerodynamic behaviour under wind loads.

Traditionally, the characterisation of aerodynamic wind action and its effects on flexible structures have been based on physical models tested in wind tunnels. More recently, an alternative numerical approach has been developed and refined [1, 2]. This empirical theory, based on the so-called Scanlan model for the evaluation of wind forces also called aeroelastic forces, involves important simplifications. However, this numerical approach requires the identification of several coefficients whose estimation assumes then central importance in the evaluation of the response of long-span bridges to wind loading. These aerodynamic coefficients strongly depend on the bridge cross section and on a particular dimensionless velocity (reduced velocity).

On the other hand, cable stayed bridge girders with two I-beams have been adopted in long span bridges because their structural and economical advantages. However, this basic $\pi$ cross section does not necessarily have good aerodynamic stability. As it can be seen in figures 1-3, the vortex located below the cross section is the main source of the generated problems. It moves from the middle close to the right side describing an ellipse in an anti-clockwise way. The moved mass and the corresponding velocity are the important features that can manage the oscillation of the aerodynamic forces, i.e. their amplitude and frequency depend on it.

In general, depending on the bridges' characteristics, some aerodynamic or structural measures against wind-induced vibrations can be done. Fairings, flaps, edge plates, side plates, baffle plates or gratings are examples of the techniques usually used to aerodynamically improve several cross sections. These attachments can be added to the structure during the construction or after that. Active or passive control of aerodynamic characteristics is also usually used as an aerodynamic stability solution. In the present case of a $\pi$ cross section, the authors have already studied the possibility of improving this typical cross section by adding baffle pates [3]. The idea consisted in breaking the anti-clockwise path of the mentioned vortex. But, taking into account the flutter phenomenon case, the results obtained suggest that the addition of baffle plates to a $\pi$ section does not improve its aerodynamical characteristics.

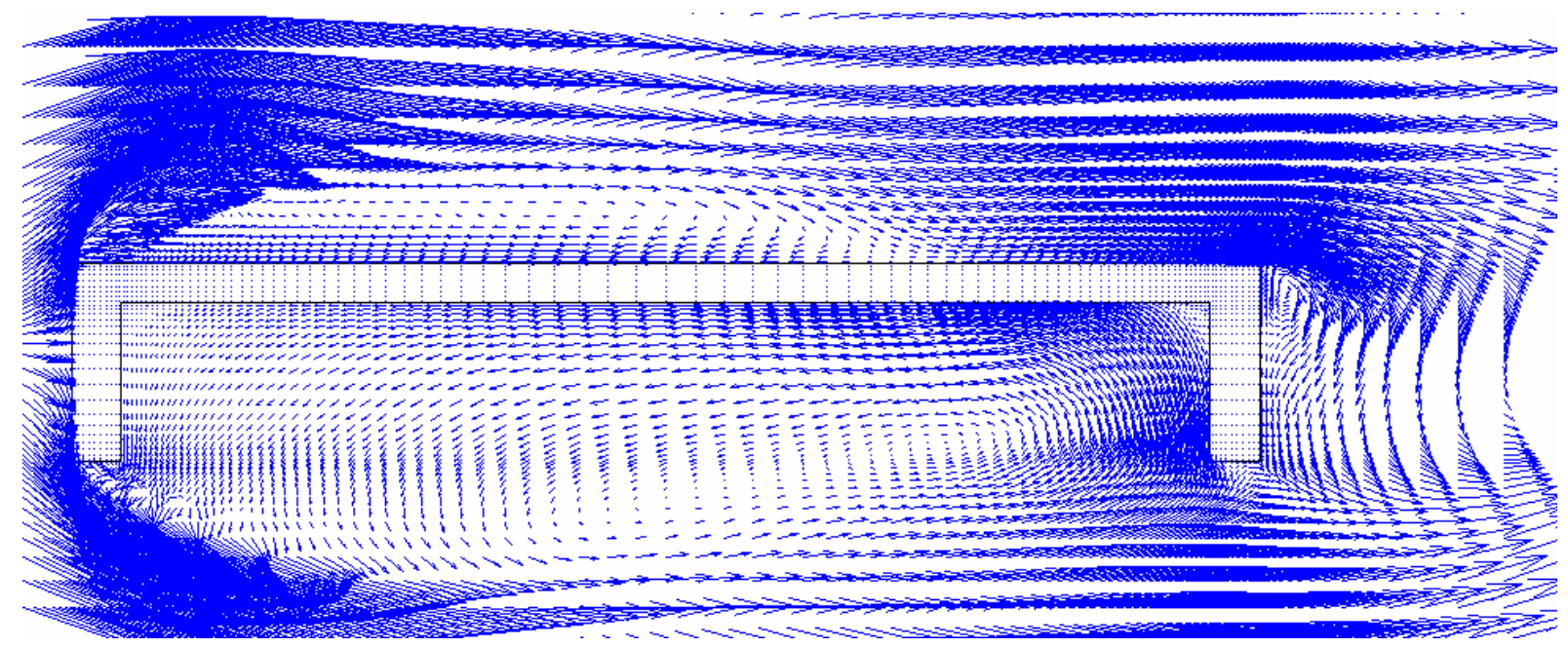

Figure 1: General velocity field around a $\pi$ cross section - stage 1. 


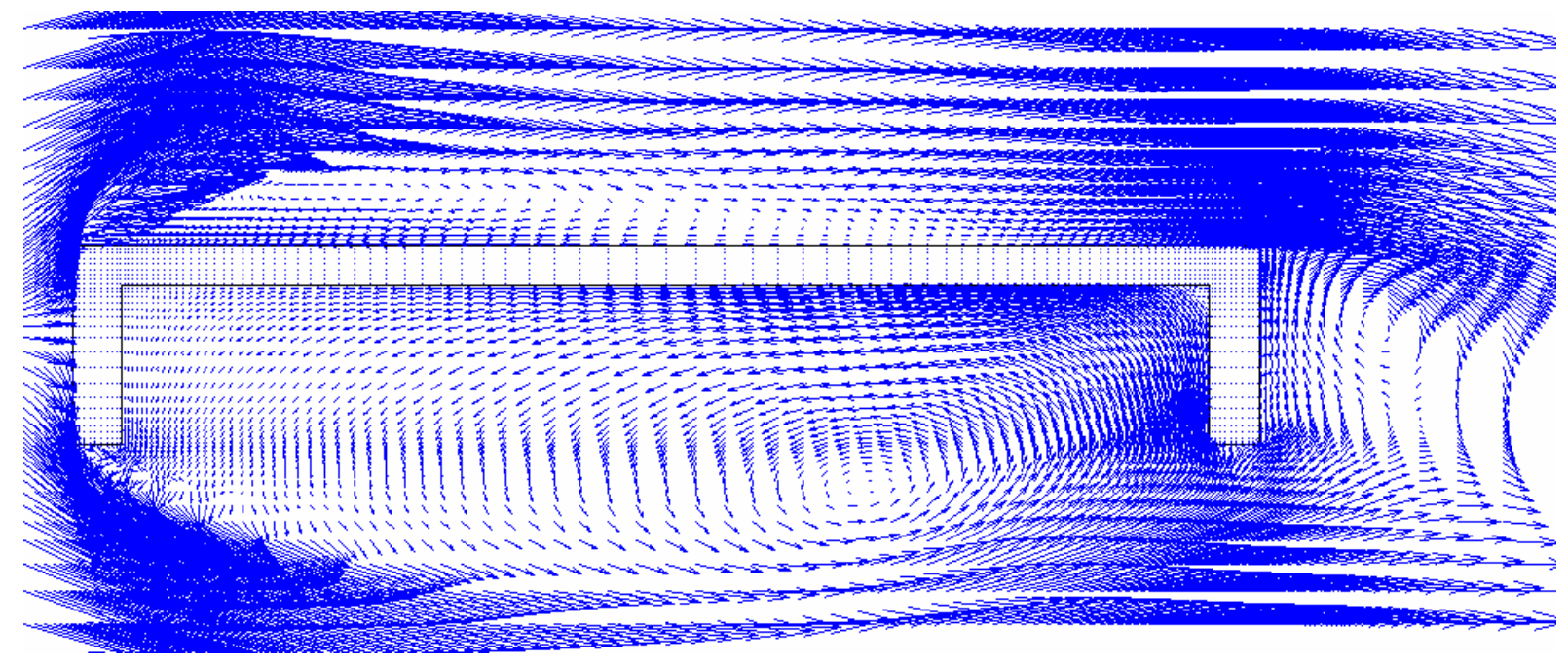

Figure 2: General velocity field around a $\pi$ cross section- stage 2.

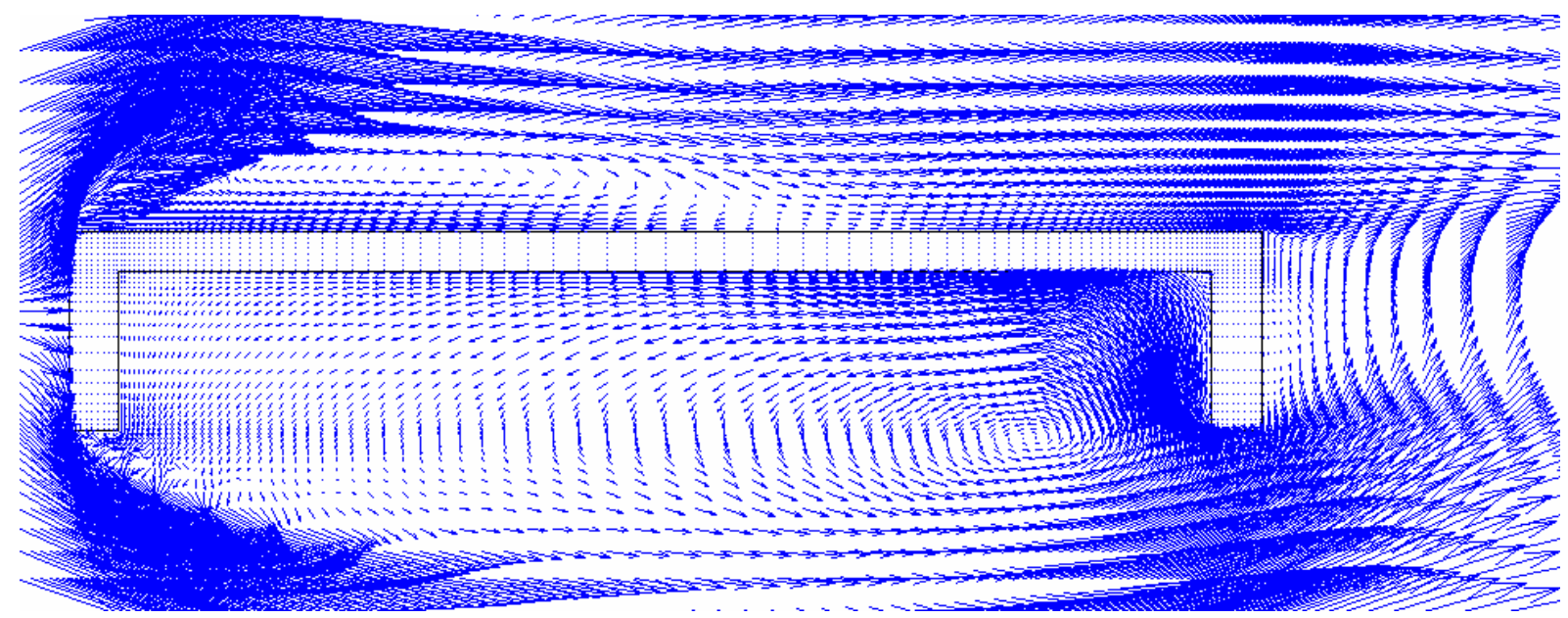

Figure 3: General velocity field around a $\pi$ cross section- stage 3.

Now, the intention of this study consists in evaluating the efficiency of using based inside plates with the purpose of improving the aerodynamic characteristics of a $\pi$ cross section. Accordingly, it is considered 5 sections: the $\pi$ section without any based lateral inside plates as a basic section, $3 \pi$ sections with based lateral inside plates of $1 \mathrm{~m}, 1.5 \mathrm{~m}$ and $2 \mathrm{~m}$ long, and the rectangular section. The based lateral inside plates are composed by two horizontal plates added to the basic section at the base of the two vertical members. In this way, the rectangular section corresponds to the limit of introducing based inside plates with long $\mathrm{B} / 2$. It is considered the Scanlan model, namely the $A_{2}^{*}$ coefficient, with the aim of evaluating the aerodynamic efficiency of the cross section. 
In order to determine the fluid flow around the obstacle, it is used a numerical algorithm of $C F D$ based on the FVM. The implemented program is suitable to simulate incompressible and isothermal bidimensional unsteady fluid flows around obstacles. It is assumed that the flow's domain may be discretised in a Cartesian and structured control volume mesh, whose faces have vertical and horizontal directions. In this algorithm, the high Reynolds number $k-\varepsilon$ turbulence diffusion model is applied to simulate the flow turbulence. All relevant equations used for modulation of fluid flow can be consulted in reference [4]. The obstacles movements were modelled indirectly by changing the velocity components of the fluid flow at external inlet boundary domain. Additionally, FOM is adopted for evaluating aeroelastic coefficients.

\section{SCANLAN MODEL}

The term "flutter" was initially used by aeronautic engineers to describe the aerodynamic instability of aircraft wings, which is characterised by both vertical and torsional oscillations. Flexible structures, such as long-span bridges, under air fluid flow action also experiment similar unstable effects. In this case, the so-called "flutter" phenomenon happens when one or more oscillating modes show increasing amplitudes due to aerodynamic forces, whose growth also depends on the structural movements. That is to say, aerodynamic forces, now called aeroelastic forces, are dependent, not only on geometry of the cross section and on velocity of the free flow, but also on the structural movements and vice-versa. In this domain, it is usual to call self-excited forces to the aeroelastic forces.

In the field of Bridge Aerodynamics, the first analytical model to explicit those aeroelastic forces was presented by Scanlan [1], with the aim of analyzing the "flutter" stability. This model considered only two degrees of freedom (one vertical and one angular), where lift and moment aeroelastic forces were dependent on rotation and its velocity, and vertical velocity. On the other hand, this model was applied under the following assumptions: $i-$ ) the free flow had no oscillation; ii-) the movements had constant frequency; iii-) and the amplitude of movements was incipient. After that, this approach was improved to complete the model [5] which is presented below considering a particular case of a cross section that has three degrees of freedom, as indicated in figure 4. In this model, it is assumed that any aeroelastic force is dependent on all movements through the displacement and velocity components.

Accordingly, the dynamic system of balanced equations can be express by

$$
\left\{\begin{array}{c}
M_{1} \cdot \ddot{a}_{1}+C_{1} \cdot \dot{a}_{1}+K_{1} \cdot a_{1}=F_{a 1} \\
M_{2} \cdot \ddot{a}_{2}+C_{2} \cdot \dot{a}_{2}+K_{2} \cdot a_{2}=F_{a 2} \\
M_{12} \cdot \ddot{a}_{12}+C_{12} \cdot \dot{a}_{12}+K_{12} \cdot a_{12}=F_{a 12}
\end{array}\right.
$$

where $M_{i}, C_{i}, K_{i}, a_{i}, \dot{a}_{i}$ and $\ddot{a}_{i}$ correspond to the mass, damping, stiffness, displacement, velocity and acceleration of cross section according to direction $i$.

Before progressing, it is worth mentioning two important dimensionless parameters: reduced velocity $U r$ and reduced frequency $K r$, defined by

$$
\begin{gathered}
U r=\frac{U}{f B}=\frac{2 \pi}{K r} \\
K r=\frac{B \omega}{U}
\end{gathered}
$$


where $U$ is the velocity of the free fluid flow and $\omega=2 \pi f$ represents the angular frequency of the system oscillation. Dimension $B$ is indicated in figure 4 .

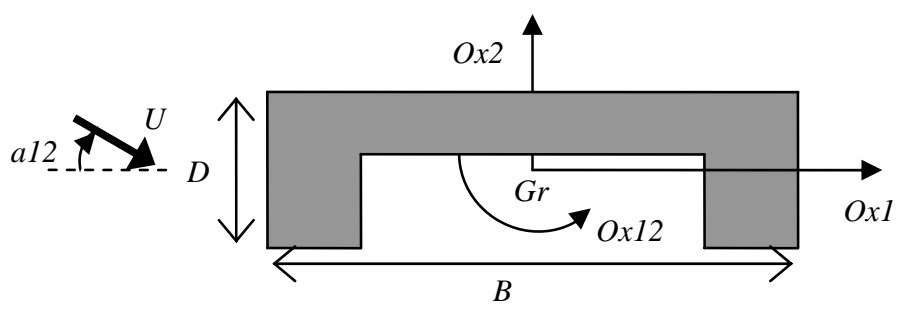

Figure 4: Degrees of freedom of a cross section.

According to the Scanlan model, the characterisation of the aeroelastic force $F_{a}$ is made by means of some constants, by aeroelastic coefficients and by movements of the cross section $a$, i.e, taking into consideration the definition of force coefficients it is possible to write down

$$
\begin{gathered}
F_{a}=\left[\begin{array}{c}
F_{a 1} \\
F_{a 2} \\
F_{a 12}
\end{array}\right]=C F \cdot P H A^{*} \cdot a \\
C F=\frac{\rho U^{2} B}{2}\left[\begin{array}{lll}
1 & 0 & 0 \\
0 & 1 & 0 \\
0 & 0 & B
\end{array}\right]
\end{gathered}
$$

where $C F$ is a matrix of force coefficient constants, $\rho$ represents the density of the fluid, and

$$
\begin{aligned}
& P H A^{*}= {\left[\begin{array}{cccccc}
\frac{K r P_{5}^{*}}{U} & \frac{K r B P_{2}^{*}}{U} & K r^{2} P_{3}^{*} & \frac{K r^{2} P_{6}^{*}}{B} & \frac{K r P_{1}^{*}}{U} & \frac{K r^{2} P_{4}^{*}}{B} \\
\frac{K r H_{1}^{*}}{U} & \frac{K r B H_{2}^{*}}{U} & K r^{2} H_{3}^{*} & \frac{K r^{2} H_{4}^{*}}{B} & \frac{K r H_{5}^{*}}{U} & \frac{K r^{2} H_{6}^{*}}{B} \\
\frac{K r A_{1}^{*}}{U} & \frac{K r B A_{2}^{*}}{U} & K r^{2} A_{3}^{*} & \frac{K r^{2} A_{4}^{*}}{B} & \frac{K r A_{5}^{*}}{U} & \frac{K r^{2} A_{6}^{*}}{B}
\end{array}\right] } \\
& a^{T}=\left[\begin{array}{llllll}
\dot{a}_{2} & \dot{a}_{12} & a_{12} & a_{2} & \dot{a}_{1} & a_{1}
\end{array}\right]
\end{aligned}
$$

and the coefficients $P_{i}^{*}, H_{i}^{*}$ e $A_{i}^{*}$ are the aeroelastic coefficients, also called Scanlan coefficients or aerodynamic derivatives. Usually, these aeroelastic coefficients are presented as a function of only two factors: geometry of cross section and reduced velocity (or reduced frequency). It is said that this linear model is valid only for incipient amplitude of cross section movements, and also only when the frequency of oscillation is quite far from the Strouhal frequency. It is also known that the incoming flow and the amplitude of oscillation are very important on the evaluation of these coefficients in the experimental field, as well as in the methodology used to get them (forced oscillation or free oscillation methods). 
Based on Fourier transform, the transformation of Scanlan equation (4-5) to frequency domain can lead to

$$
\begin{aligned}
& \tilde{F}_{a 1}=\frac{\rho U^{2} B K r^{2}}{2}\left[\left(P_{4}^{*}+i P_{1}^{*}\right) \frac{\tilde{a}_{1}}{B}+\left(P_{3}^{*}+i P_{2}^{*}\right) \tilde{a}_{12}+\left(P_{6}^{*}+i P_{5}^{*}\right) \frac{\tilde{a}_{2}}{B}\right] \\
& \tilde{F}_{a 2}=\frac{\rho U^{2} B K r^{2}}{2}\left[\left(H_{4}^{*}+i H_{1}^{*}\right) \frac{\tilde{a}_{2}}{B}+\left(H_{3}^{*}+i H_{2}^{*}\right) \tilde{a}_{12}+\left(H_{6}^{*}+i H_{5}^{*}\right) \frac{\tilde{a}_{1}}{B}\right] \\
& \tilde{F}_{a 12}=\frac{\rho U^{2} B^{2} K r^{2}}{2}\left[\left(A_{4}^{*}+i A_{1}^{*}\right) \frac{\tilde{a}_{2}}{B}+\left(A_{3}^{*}+i A_{2}^{*}\right) \tilde{a}_{12}+\left(A_{6}^{*}+i A_{5}^{*}\right) \frac{\tilde{a}_{1}}{B}\right]
\end{aligned}
$$

where $\tilde{F}_{i}$ and $\tilde{a}_{i}$ correspond to the Fourier transform of aeroelastic forces and movements according to direction $i$. That means that, if all parameters are known or specified, and if it is calculated both the Fourier transform of movements $\tilde{a}_{i}$ according to a particular direction $i$ and the Fourier transforms of all aeroelastic forces $\tilde{F}_{i}$, then the correspondent aeroelastic coefficients can be evaluated using the last three equations.

For instance, on FOM context, used by Nakamura [6], the movements according to a particular direction $i$ are imposed, so it is only needed the calculation of the three aeroelastic forces and the subsequent Fourier transforms to evaluate the six matching Scanlan coefficients.

The displacement $a=a_{0} \cos (\omega t)$ and the velocity $\dot{a}=-a_{0} \omega \sin (\omega t)$ imposed to a cross section were defined by Nakamura as sinusoidal functions with constant amplitude $a_{0}$ and constant frequency $\omega$. The symbol $t$ represents the time.

\section{NUMERICAL PROCEDURES}

To evaluate the efficiency of using based lateral inside plates with the purpose of improving the aerodynamic characteristics of a $\pi$ cross section $(B / D=6)$, five different cross sections were used (see figure 5): the $\pi$ section without any based lateral inside plates (S1) as a basic section, $3 \pi$ sections with based lateral inside plates of $1.0 \mathrm{~m}$ (S2), $1.5 \mathrm{~m}$ (S3) and 2.0m (S4) long, and the rectangular section (S5). It is considered a structured control volume mesh whose main characteristics of the domain and the corresponding discretisation, according to figure 6 , are: $B=12 \mathrm{~m} ; D=2 \mathrm{~m} ; L 1=19.8 \mathrm{~m} ; L 2=15.0 \mathrm{~m} ; L 3=31.8 \mathrm{~m}$; maximum dimension $=750 \mathrm{~mm}$; minimum dimension $=51 \mathrm{~mm}$. The numbers of the Control Volumes used are from 81 to 89 for direction 2, and 167 to 227 for direction 1.

Usually, aeroelastic coefficients are presented graphically conditional on reduced velocity $U r$. Accordingly, for each graph, it is used 9 points, from $U r=1.0$ to $U r=15$.

It is considered two velocities of the free fluid (air at standard conditions) flow: $U=0.376 \mathrm{~m} / \mathrm{s}(R e=5 E 4)$ and $U=37.6 \mathrm{~m} / \mathrm{s}(R e=5 E 6)$. The used time increment were $\Delta t=2 E-2 \mathrm{~s}$ and $\Delta t=2 E-4 s$ respectively. For each case, table 1 shows some static relevant values, where $C F i$ represents the average aerodynamic force coefficient according to direction $i, \Delta$ means the average amplitude of variation, and St corresponds to the Strouhal number. The time interval is established in order to get stability during the simulation, as much as necessary. 

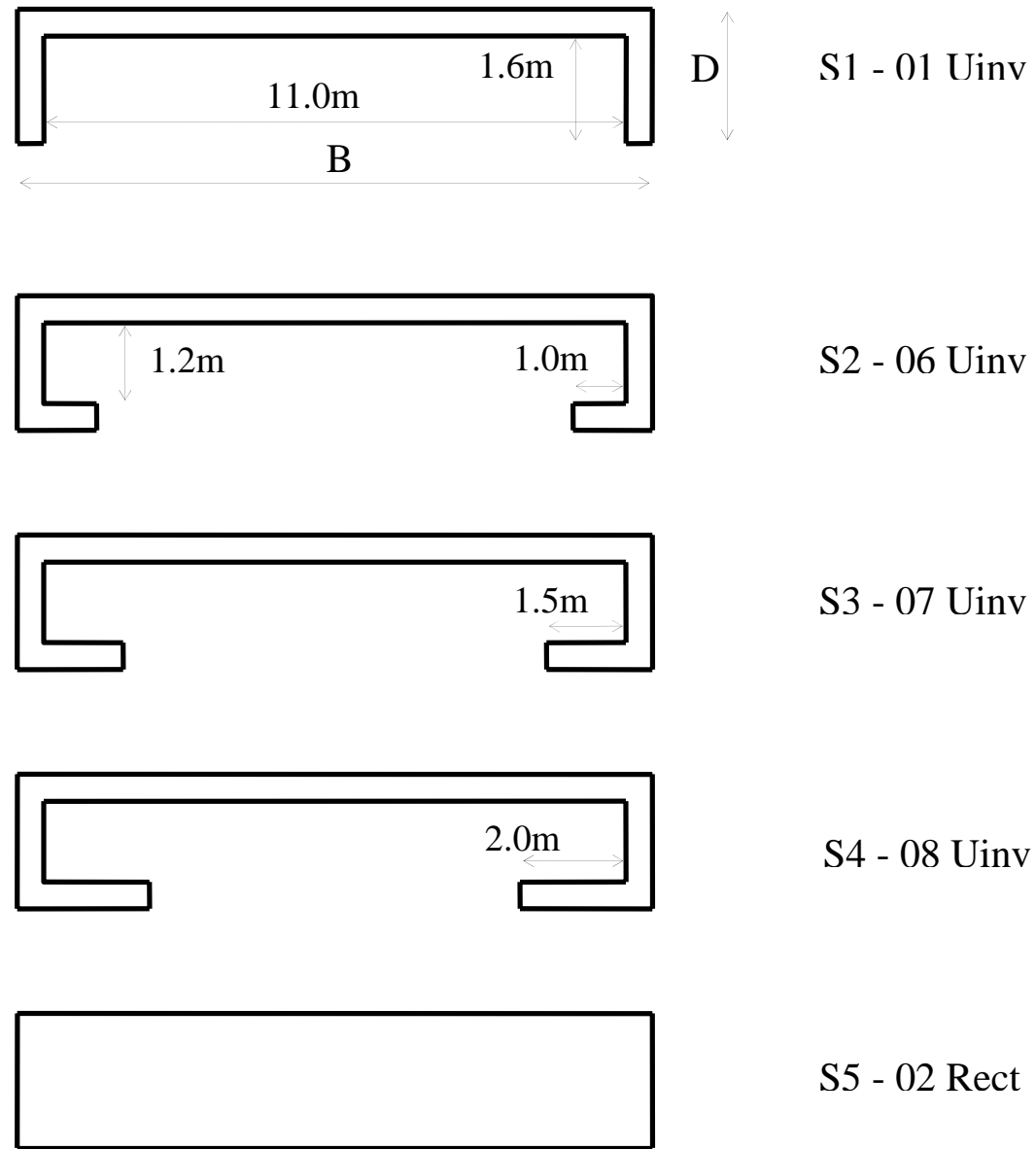

S5 - 02 Rect

Figure 5: Five cross sections considered.

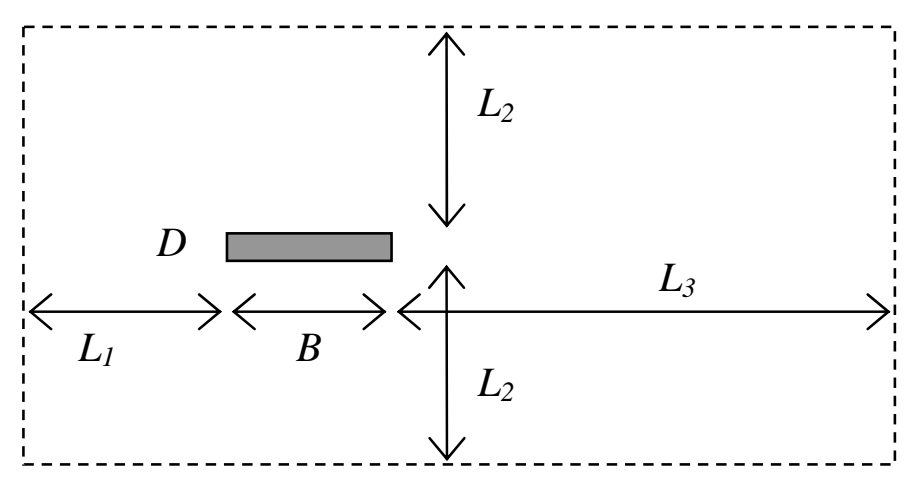

Figure 6: Main characteristics of the domain. 


\begin{tabular}{|c|c|c|c|c|c|c|c|c|}
\hline & & CF1 & $\triangle C F 1$ & CF2 & $\triangle C F 2$ & CF12 & $\triangle C F 12$ & St \\
\hline \multirow{2}{*}{ S1 } & $R e=5 E 4$ & 1.181 & 0.332 & -0.428 & 0.236 & -0.239 & 0.174 & 0.120 \\
\hline & $R e=5 E 6$ & 1.217 & 0.272 & -0.411 & 0.197 & -0.292 & 0.168 & 0.124 \\
\hline \multirow{2}{*}{$S 2$} & $R e=5 E 4$ & 1.128 & 0.347 & -0.268 & 0.199 & -0.104 & 0.118 & 0.124 \\
\hline & $R e=5 E 6$ & 1.215 & 0.277 & -0.300 & 0.186 & -0.168 & 0.135 & 0.126 \\
\hline \multirow{2}{*}{ S3 } & $R e=5 E 4$ & 1.127 & 0.320 & -0.177 & 0.190 & -0.040 & 0.108 & 0.126 \\
\hline & $R e=5 E 6$ & 1.202 & 0.249 & -0.236 & 0.172 & -0.120 & 0.116 & 0.126 \\
\hline \multirow{2}{*}{$S 4$} & $R e=5 E 4$ & 1.110 & 0.287 & -0.127 & 0.183 & 0.007 & 0.109 & 0.126 \\
\hline & $R e=5 E 6$ & 1.183 & 0.214 & -0.185 & 0.158 & -0.079 & 0.103 & 0.126 \\
\hline \multirow{2}{*}{ S5 } & $R e=5 E 4$ & 1.136 & 0.038 & Residual & 0.120 & Residual & 0.131 & 0.104 \\
\hline & $R e=5 E 6$ & 1.095 & 0.002 & Residual & 0.044 & Residual & 0.027 & 0.116 \\
\hline
\end{tabular}

Table 1: Some relevant values got when the cross section is at rest.

Static results permit to draw some initial conclusions: firstly, the results obtained for the different cross sections are quite different and they are closely associated with the separationand-reattaching flow and the vortex development between based lateral inside plates; secondly, as the velocity of free fluid flow grows, the amplitude oscillation of aerodynamic forces becomes lower and lower; thirdly, one can understand that based lateral inside plates limit is the rectangular cross section.

In figures 5-7 are shown the general velocity fields of the flow around sections S2, S3 and S4. As evaluated, the vortex located at the inside plates moves lesser as the width of the inside plates increases. But, it should be pointed out that near downstream additional plate there is a field with a higher velocity, i.e. a higher dynamic pressure, but with small amplitude of oscillation.

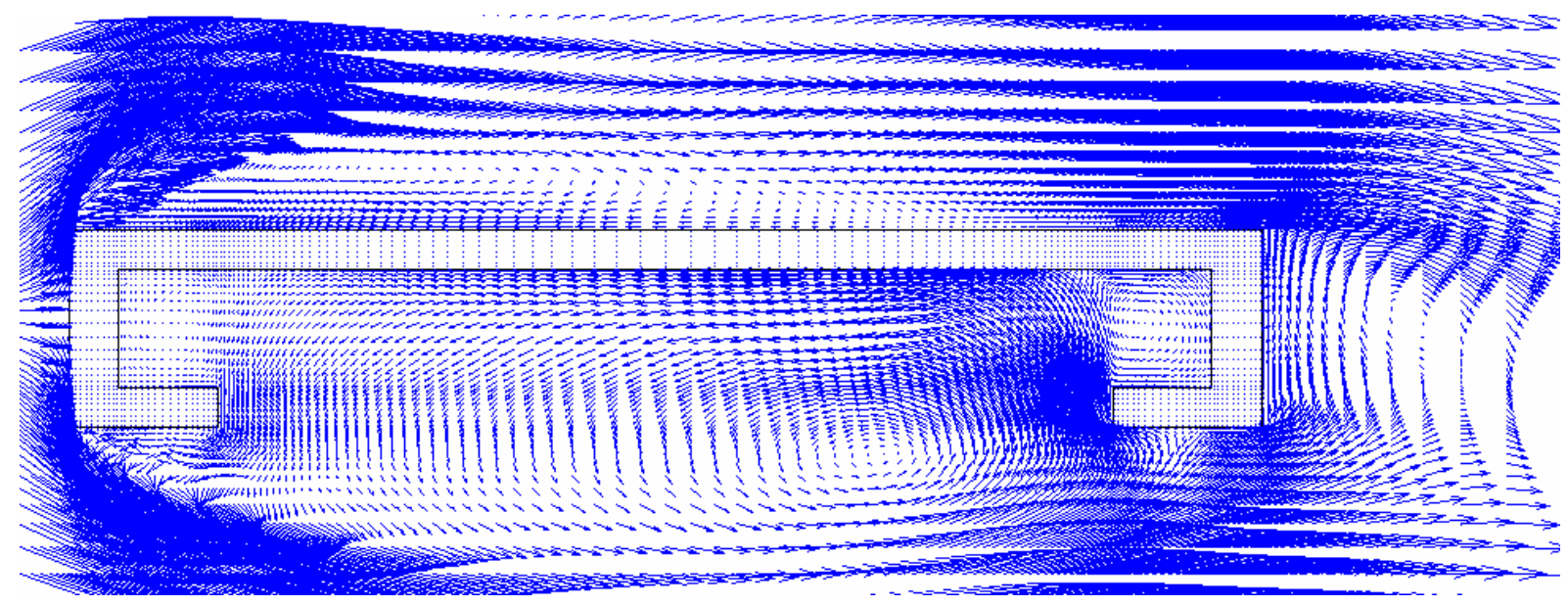

Figure 7: General velocity field around S2 cross section. 


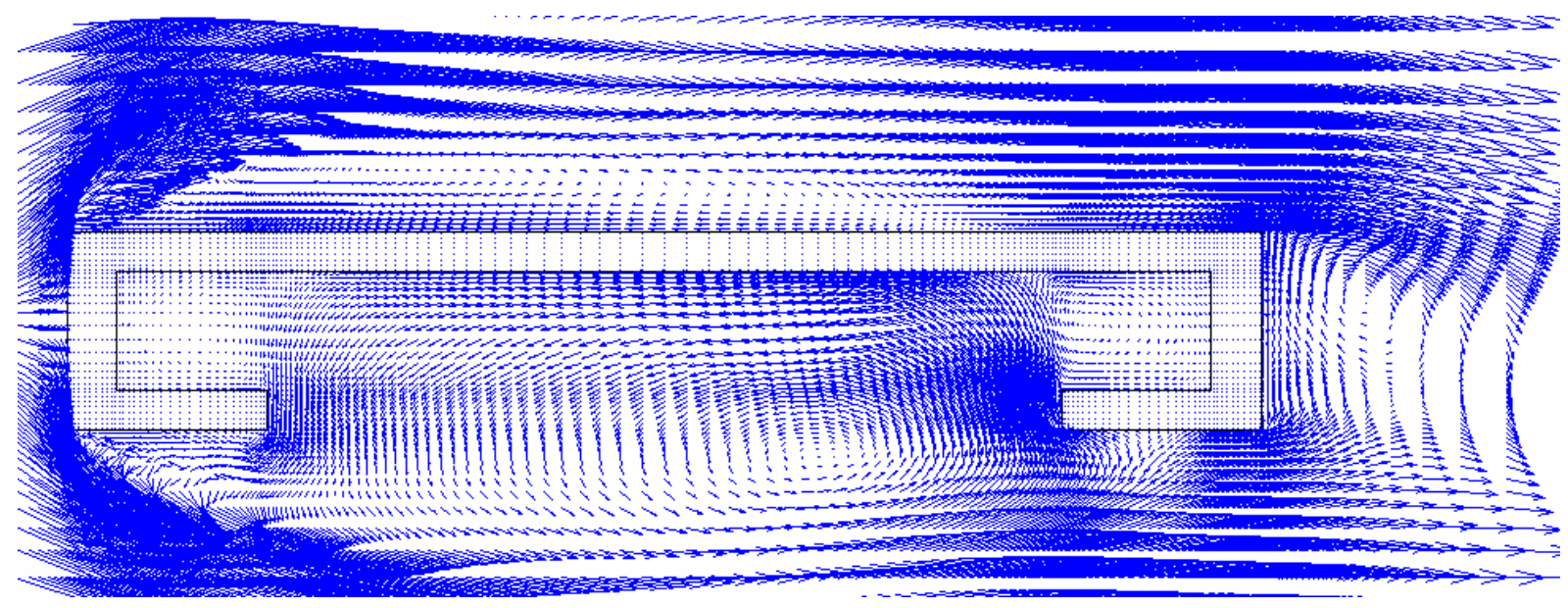

Figure 8: General velocity field around S3 cross section.

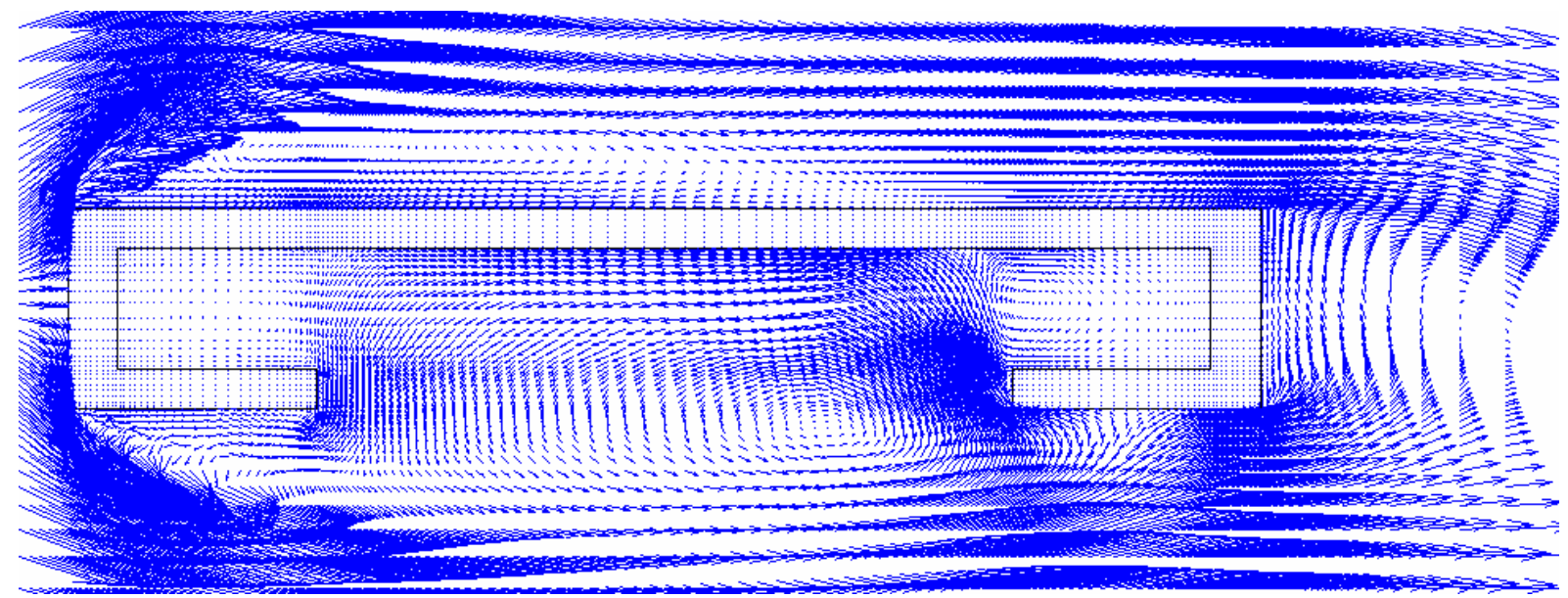

Figure 9: General velocity field around S4 cross section.

Like it was evaluated, the amplitude of aerodynamic forces decreases mainly due to the movement restraining of the inside vortex. This restraining is the direct consequence of the inside plates' width. At the limit, i.e. for the rectangular cross section, the amplitude of aerodynamic forces depend only on the vortex shedding in the wake, once that the above and below vortices are relatively stables, mainly because the separated flow reattaches before the wake.

However, more aeroelastic stability is not a consequence of small amplitude of aerodynamic forces obtained considering fixed obstacle. In fact, for instance, and as it was shown before [3], the rectangular section has inferior values of $\triangle C F 2$ and $\triangle C F 12$, when compared with the $\pi$ section, but, at least, the flutter coefficient is higher, i.e. the rectangular section is more unstable than the $\pi$ section from the aeroelastic point of view.

Considering the evolution of Scanlan coefficients and taking into consideration the amplitude of forced oscillation, two important aspects mentioned in the literature $[2,6]$ should be referred: firstly, the Scanlan model is valid only for incipient amplitude oscillations of the cross section; secondly, this amplitude is usually dimensionless by means of $D$, with values inferior to 0.1 . However, taking into consideration the physical aspect revealed by equations (8-10), it is possible to conclude that, for forced rotations, the amplitude of displacement is the key parameter to state the changing conditions, but for forced translations, the important 
parameter is the velocity of forced movement, not the displacement. So, in this work, the amplitude of forced rotations is assumed as $a_{0}=3 \%(\mathrm{rad})=1.72^{\circ}$ while, for forced translational cases, with an angular frequency $w, a_{0} * w \approx 3 \% * U \Rightarrow a_{0}=0.005 B * U r$. Last statement means that the maximum velocity of forced oscillation is limited to $3 \%$ of the flow velocity.

Each simulation has four phases to be completed. In the first one, the velocity of the free flow is increased to reach the desired value keeping the cross section fixed. Next, the time step is adapted if necessary. The objective consists in having an appropriate discretisation (not less than 500 time steps) of the forced oscillation period. In the third phase, it is expected that the corresponding aeroelastic forces reach a regular pattern of oscillation. At last, it is made the record for evaluation of the correspondent aeroelastic coefficients. The record must have more than 20000 time steps, or at least 5 forced oscillation periods.

\section{SCANLAN COEFFICIENTS EVALUATION}

This work has produced a large amount of results, from which it is only possible to present the most important graphs which support the conclusions written down below.

In order to inspect the dependence of the baffle plate's number on the evaluated values of the aeroelastic coefficients, it is presented in figures (10-13) the most important aeroelastic coefficients $H_{1}^{*}$ and $A_{2}^{*}$ used for calculation of vertical and torsional stability.

With the intention of confronting the current results, it is presented in figures (14-15) the results obtained experimentally by Matsumoto [7] and by this methodology considering rectangular sections. The dimensions considered are $B / D=6$, in this study, and $B / D=5$ and $B / D=8$ for the Matsumoto cases.

Just to be shown, figures 16-19 present the remaining important aeroelastic coefficients related to the $\pi$ section, considering the two simulated flows.

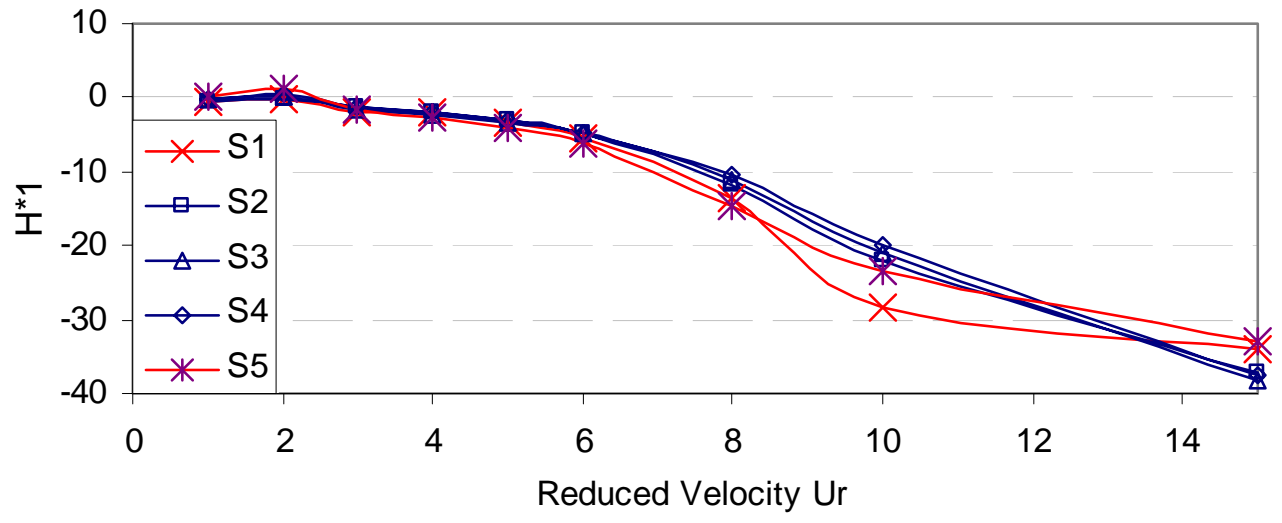

Figure 10: Aeroelastic coefficient $H_{1}^{*}$ evaluated for five different sections ( $\left.\mathrm{Re}=5 \mathrm{E} 4\right)$. 


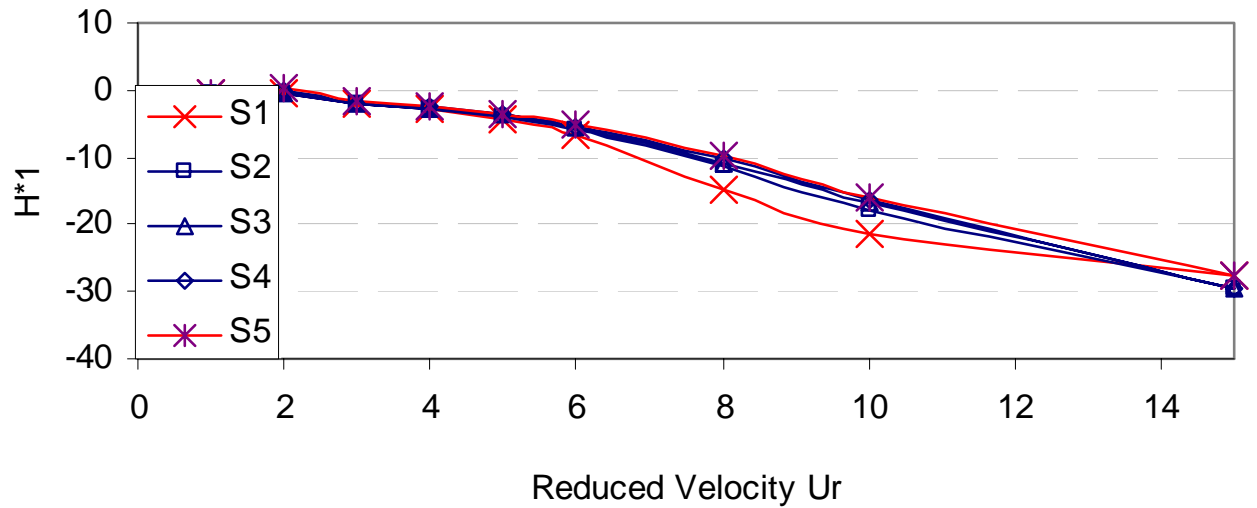

Figure 11: Aeroelastic coefficient $H_{1}^{*}$ evaluated for five different sections (Re=5E6).

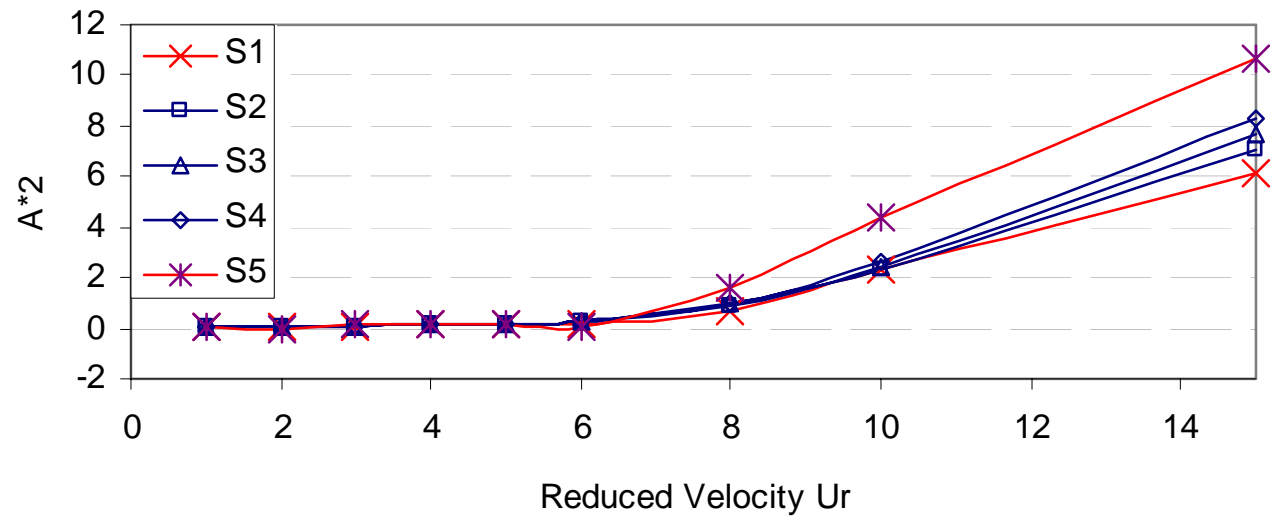

Figure 12: Aeroelastic coefficient $A_{2}^{*}$ evaluated for five different sections (Re=5E4).

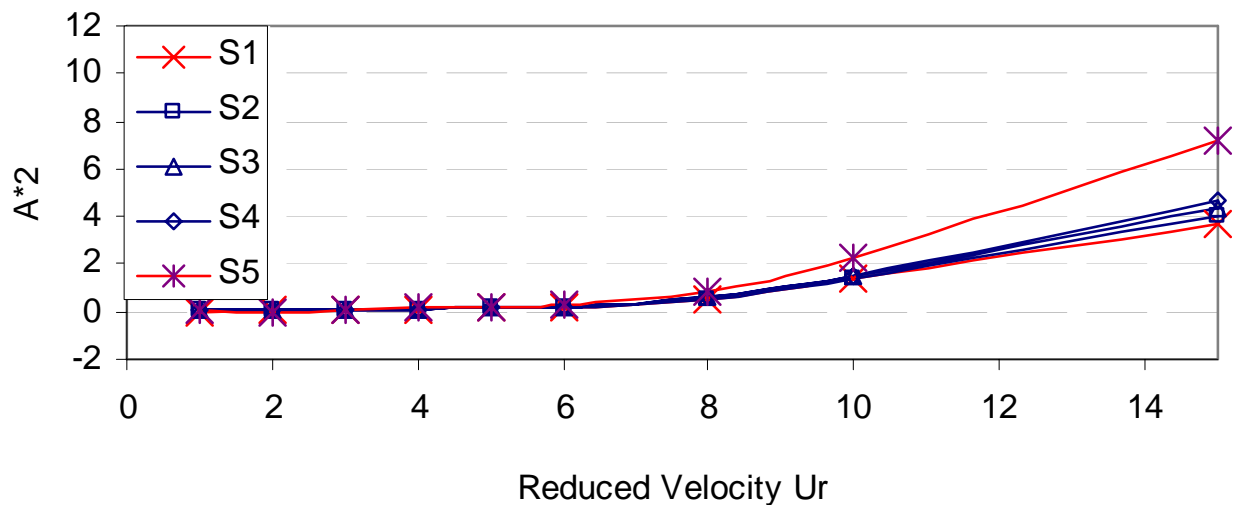

Figure 13: Aeroelastic coefficient $A_{2}^{*}$ evaluated for five different sections (Re=5E6). 


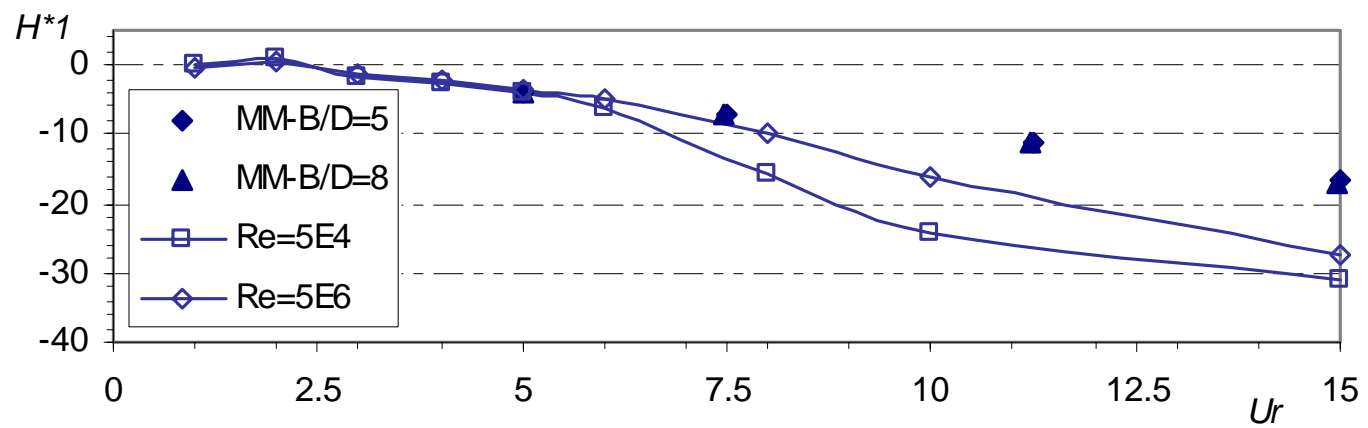

Figure 14: Comparing aeroelastic coefficient $H_{1}^{*}$ evaluated for rectangular sections.

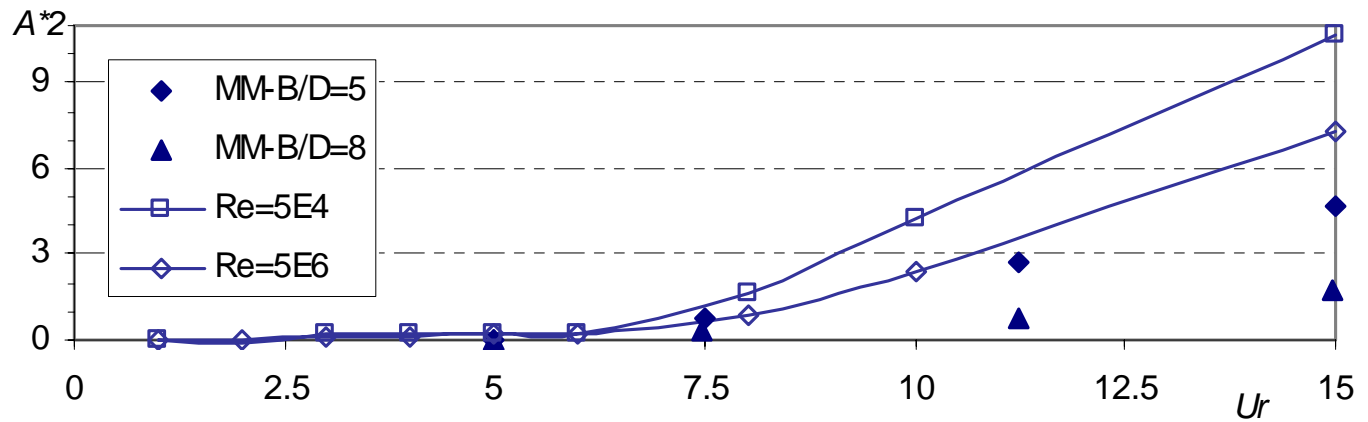

Figure 15: Comparing aeroelastic coefficient $A_{2}^{*}$ evaluated for rectangular sections.

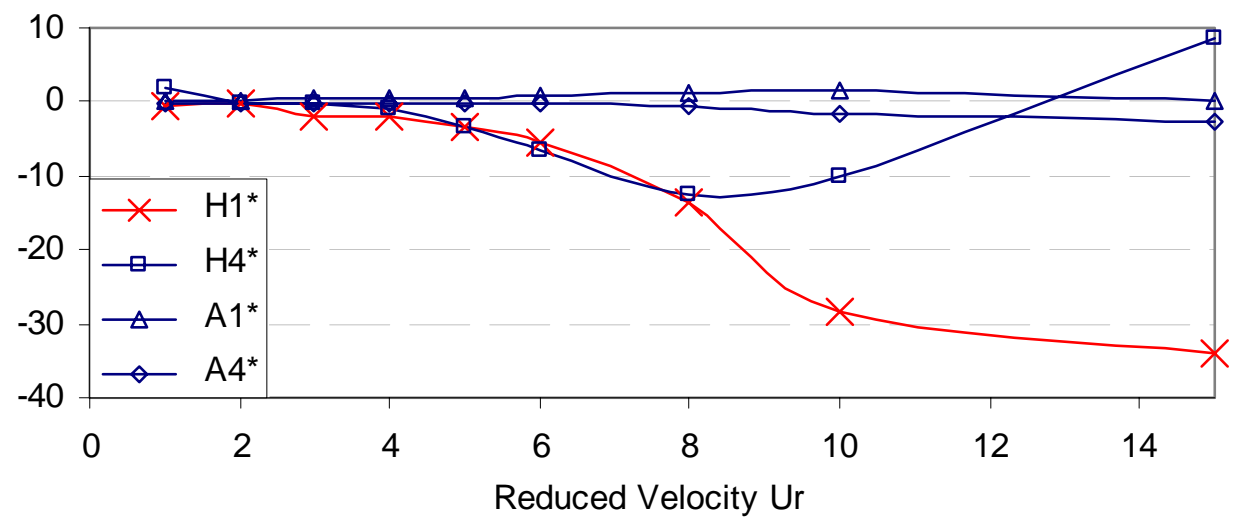

Figure 16: Aeroelastic coefficients evaluated for the $\pi$ section. Vertical forced oscillations. Re=5E4. 


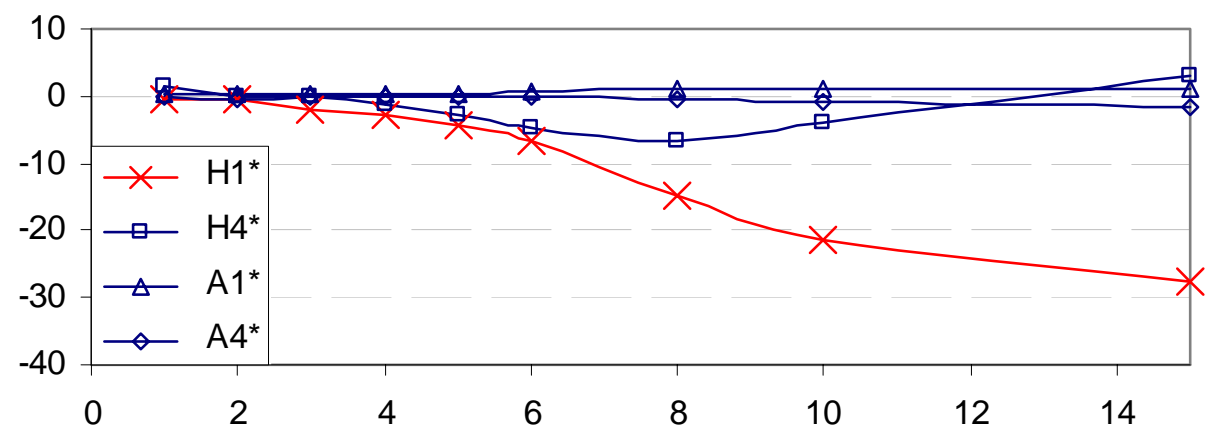

Reduced Velocity Ur

Figure 17: Aeroelastic coefficients evaluated for the $\pi$ section. Vertical forced oscillations. Re=5E6

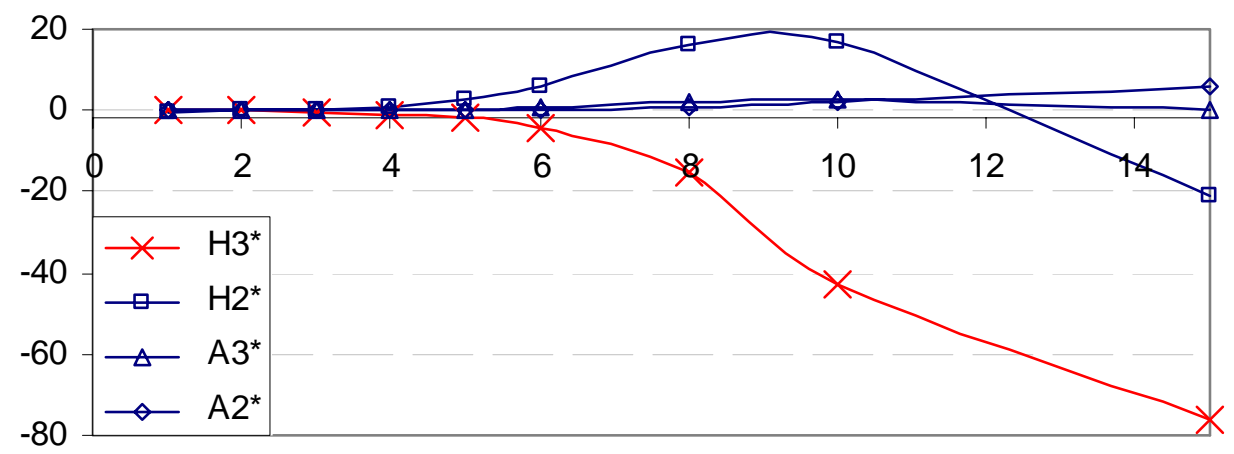

Reduced Velocity Ur

Figure 18: Aeroelastic coefficients evaluated for the $\pi$ section. Angular forced oscillations. Re=5E4.

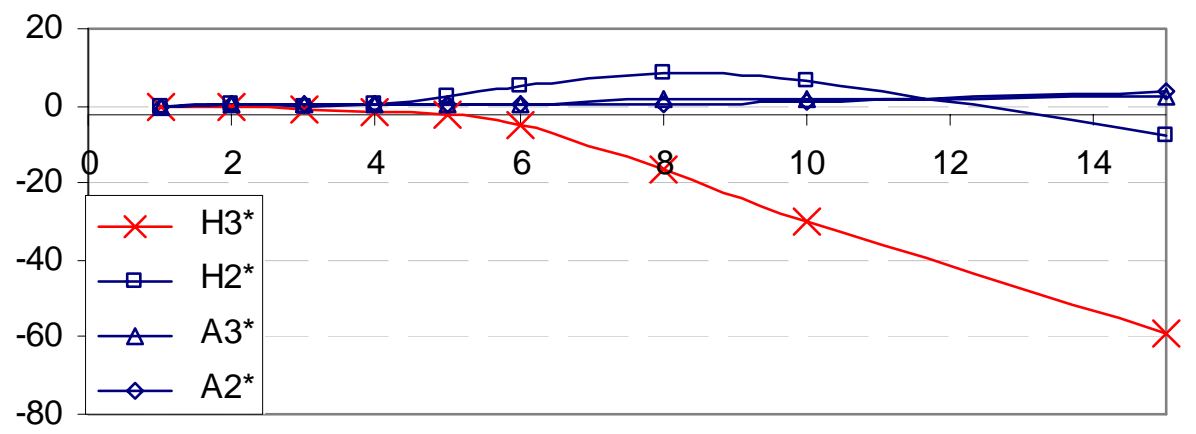

Reduced Velocity Ur

Figure 19: Aeroelastic coefficients evaluated for the $\pi$ section. Angular forced oscillations. Re=5E6.

The inspection of these results permit to draw the following particular conclusions, considering the aeroelastic coefficients $H_{1}^{*}$ and $A_{2}^{*}$ :

- the aeroelastic coefficients evaluated when considering moderate velocities $(R e=5 E 4)$ diverge from those obtained when considering high convective flows $(R e=5 E 6)$ (see figures 10-13); 
- in particular, for the basic section $S 1$, the aeroelastic coefficient $A_{2}^{*}$ changes from 2.35 ( $R e=5 E 4)$ to $1.40(R e=5 E 6)$ when reduced velocity stands for 10 (see figures 12-13);

- for example, considering one structure characterised by $f=0.5 \mathrm{~Hz}$ and with the stability limit of $A_{2}^{*}=2$, this could mean a critical flutter velocity of $28.8 \mathrm{~m} / \mathrm{s}(U r=9.6$ for $R e=5 E 4$ case) or $34.2 \mathrm{~m} / \mathrm{s}$ ( $U r=11.4$ for $R e=5 E 6$ case), which represents a significant difference;

- for aeroelastic coefficient $H_{1}^{*}$, the differences evaluated for the five cross sections are not so important as the corresponding values are negative, i.e. they work for stability (see figures 10-11);

- for all the considered five sections, the torsional instability, i.e. the flutter phenomenon, will be less probable to happen for reduced velocities lower than 5 , as the aeroelastic coefficient $A_{2}^{*}$ assumes quasi null values in this range;

- on the other hand, when the reduced velocity is high, the most important aeroelastic coefficient $A_{2}^{*}$ have significant values (see figures 12-13);

- taking into consideration only the changing of the aeroelastic coefficient $A_{2}^{*}$, one can bring up that the basic $\pi$ section gets close to the rectangular section as the width based lateral inside plates increases;

- considering only the possibility of torsional instability, i.e. the evaluation of aeroelastic coefficient $A_{2}^{*}$, figures 12 and 13 show that the basic $\pi$ section is also the less sensitive to flutter of the considered five sections;

- the addition of based lateral inside plates can restrain the vortex movement when the section is considered fixed. Even though, and considering the forced movements' context, unfortunately, the oscillation of aeroelastic forces has higher amplitudes, becoming this section more unstable from the flutter point of view;

- taking into consideration the values obtained by Matsumoto (see figures 14-15), it is possible to conclude that there is a poor agreement for $R e=5 E 4$ case, which is close to the velocity used by Matsumoto. Even, for $\mathrm{Re}=5 \mathrm{E} 6$ case, the corresponding results are not close, mainly for high reduced velocities range.

\section{CONCLUSIONS}

For evaluating the effects of wind action on flexible structures such as long-span bridges, it is generally used a numerical approach based on the so called Scanlan model, which requires the identification of some aeroelastic coefficients. The objective of this paper is to present a numerical study associated with the efficiency of using based lateral inside plates with the purpose of improving the aerodynamic characteristics of a $\pi$ cross section $(B / D=6)$.

The results presented here are evaluated by a numerical approach based on an algorithm of CFD (FVM). The FOM is the methodology used for numerical evaluation of aeroelastic coefficients. The computer code developed on the basis of this methodology is applied to the aeroelastic study of five cross-sections, which permits to characterise the influence of the width of based lateral inside plates on the evaluation of the most important aeroelastic coefficients. 
Considering the variation of the most important aeroelastic coefficients used for the analysis of vertical and torsional stability, one may essentially conclude that:

- concerning vertical oscillations, the basic $\pi$ section, including or not based lateral inside plates, and the rectangular section are all stables;

- taking into account the flutter phenomenon case, the results obtained suggest that the addition of based lateral inside plates to a $\pi$ section does not improve its aerodynamical characteristics;

- the evaluation of the aeroelastic coefficients, namely $A_{2}^{*}$, depends on the velocity of the free flow considered.

Under these circumstances, it seems important to have specific rules in terms of the characteristics of incoming fluid flow in order to evaluate this important aeroelastic coefficient, whose values are determinant for the evaluation of the critical velocity of aeroelastic instability.

The conclusions drawn from the specific case of the $\pi$ section can not be directly extrapolated to other examples, although the methodology presented in this paper can be applied to other cases with different shapes of cross section.

\section{REFERENCES}

[1] Scanlan, R. H. \& Tomko J. J., Airfoil and Bridge deck flutter derivatives. Journal of the Engineering Mechanics Division, ASCE, 97(6), pp. 1717-1737, 1971.

[2] Simiu E. \& Scanlan, R., Wind effects on structures. An introduction to wind engineering. John Wiley \& Sons, 1986.

[3] Diogo G., A. V. Lopes \& L. M. C. Simões, CFD based numerical study on aerodynamic characteristics of $\pi$ cross sections using baffle plates, Third International Conference on Fluid Structure Interaction - FSI2005, La Corunna, Spain, 19-21 September 2005.

[4] Lopes, A. V., Aplicação da Dinâmica Computacional de Fluidos à Análise Aeroelástica de Estruturas Esbeltas. PhD. Thesis, Departamento de Eng. Civil, FCTUC, Coimbra, Portugal, 2001.

[5] Jones, N. P, \& Scanlan, R. H., Advances (and challenges) in the prediction of long-span response to wind. Proceedings of the international symposium on advances in bridge aerodynamics, Copenhagen, Denmark, pp. 59-85, A. A. Balkema, Rotterdam, Netherlands, 1998.

[6] Nakamura, Y. \& Mizota T., Torsional flutter of rectangular prisms. Journal of the Engineering Mechanics Division, ASCE, 101(2), pp. 125-142, 1975.

[7] Matsumoto, M., Aerodynamic damping of prisms. Journal of Wind Engineering and Industrial Aerodynamics, Elsevier, 59, pp. 159-175, 1996. 